\title{
Appliquer les catégories d'observation discursives pour décrire les interactions dans les forums de discussion
}

\author{
Applying discourse observation categories for analyzing \\ online interaction in public internet forum
}

\author{
Joanna Górecka \\ Université Adam Mickiewicz de Poznań \\ jgorecka@amu.edu.pl
}

\begin{abstract}
The paper presents the objectives, the scenario and the conclusions of an exploratory action-research whose aim was to determine how the descriptive categories elaborated in the field of discourse analysis can be used to explain and to explore the genre of online discussion in public internet forums in the context of foreign language learning. I discuss first a list of descriptive categories elaborated in my research in the form of an observation grid in order to outline the genre specificity of online discussion. Then I describe the application of the observation grid in my research.
\end{abstract}

Keywords: language learning; online discussion; public internet forums; genre; discourse analysis

\section{INTRODUCTION}

Le présent travail explore les avantages - pour les apprenants de langue - de la participation aux interactions dans les forums publics et ouverts. Lors de notre travail avec les étudiants de niveau avancé, nous avons pu constater que les dispositifs publics de discussion, tels les forums de discussion, les espaces de commentaires ouverts sur les sites d'information ou les blogues, restent une ressource peu explorée par les apprenants. Indubitablement, la consultation irrégulière s'explique en partie par la nature hétérogène de ces espaces de communication : différents objectifs qui poussent les locuteurs à prendre la parole et différentes formes des échanges qui s'y 
forment. Les étudiants reculent aussi devant les dérapages et les actes d'incivilités que l'on peut observer dans les fils de discussion, ils se plaignent également de la vacuité de certains commentaires. On est donc très loin des idéaux des années 90 où les dispositifs qui rendaient possible la communication médiée par ordinateur étaient considérés comme une vraie porte vers les contacts interculturels, permettant aux apprenants de communiquer dans des situations dites authentiques, engageant de nombreux locuteurs et portant sur des sujets très variés, répondant à leurs choix personnels.

Même si la pratique quotidienne des internautes d'aujourd'hui nous oblige à relativiser les avantages liés à la communication massive et anonyme sur Internet pour les apprenants de langue, nous restons pourtant convaincue de la valeur de l'expérience d'écriture asynchrone pour perfectionner l'expression argumentative et la capacité à comprendre et à se positionner face aux sujets d'actualité discutés dans les médias. Dans le présent travail, notre questionnement portera sur la manière dont les locuteurs intègrent la communauté discursive dans les espaces de discussion publics et sur les techniques d'enseignement pouvant faciliter cette intégration. Nous souhaitons notamment mettre en relief les catégories élaborées au sein de l'analyse du discours et qui pourraient - selon nous - être utilisées par les apprenants-locuteurs pour interpréter l'activité communicative dans le forum et pour y participer. En effet, nous sommes d'avis que le recours aux recherches entreprises dans ce domaine pour décrire la communication informelle, publique et endolingue, peut apporter des outils conceptuels permettant à l'enseignant de construire un cadre théorique et de proposer des catégories d'observation opératoires pour modéliser le genre de la discussion dans le forum et insister sur l'importance de la notion d'identité discursive. Nous avançons que cette approche permet de cerner l'efficacité communicative des locuteurs engagés dans le forum en la rapportant non seulement à leur compétence de communication (maîtrise de ressources langagières) et à leur expertise dans le domaine donné mais surtout en la liant à leur capacité à adopter l'expression en ligne aux particularités génériques de la discussion et aux normes discursives propres à la communauté virtuelle.

\section{PROBLÈME DE RECHERCHE ET MÉTHODOLOGIE}

Dans la travail présenté, nous nous sommes intéressée à la manière dont on peut adapter au contexte scolaire (FLE) certaines notions et certains schémas interprétatifs élaborés dans le cadre théorique de l'analyse du discours (Charaudeau, 1993 ; Charaudeau, Maingueneau, 2002) et utilisés ensuite dans les recherches sur la communication dans les forums dans les contextes semi-formels et endolingues (Matuszak, 2007 ; Thiault, 2009 ; Doury, Lefébure 2006 ; Doury, Maroccia, 2007 ; MourlhonDallies, 2007). Nous résumerons, dans les sections qui suivront, la démarche choisie 
dans notre recherche-action et ses principales conclusions. Nous présenterons les catégories d'observation discursives retenues pour mettre en valeur la nature et les enjeux communicatifs et cognitifs des interactions dans les forums ouverts et publics et nous illustrerons, avec les données de notre corpus, comment les éléments constitutifs de la grille proposée ont été appliqués dans notre pratique enseignante. Il faut souligner que la recherche décrite a été de nature exploratoire : en conséquence, même si l'application des catégories discursives retenues a permis, dans le cadre du cours de FLE proposé, de confirmer leur valeur explicative et illustrative, cette application nécessiteraient d'être systématisée afin d'évaluer l'outil didactique proposé de manière solide et qualifiée.

La question centrale pour notre recherche est formulée comme suit : Dans quelle mesure les catégories d'observation issues de l'analyse du discours permettent-elles de décrire l'interaction en ligne et de fournir un feedback formatif aux étudiants ?

Deux questions de recherche spécifiques ont organisé notre démarche :

a. Comment les catégories d'observation retenues illustrent-elles les particularités du nouveau genre, celui de la discussion dans les forums?

Il s'agira de présenter les catégories descriptives retenues pour une analyse visant à mettre en relief les particularités génériques de la discussion dans le forum, ainsi que la spécificité du contrat de communication censé permettre aux locuteurs de considérer aussi bien la lecture que la participation comme une expérience argumentative, où sont exprimées et élaborées des opinions individuelles par rapport aux questions débattues dans l'espace public.

b. Quelle est l'efficacité d'un travail d'observation qui porte sur l'activité interprétative et rédactionnelle des locuteurs et qui s'appuie sur les catégories retenues?

Il sera question ici de montrer comment les notions exploitées dans le champ de l'analyse du discours ont été utilisées au cours de notre recherche pour décrire l'interaction et pour expliquer les mécanismes grâce auxquels les locuteurs intègrent la communauté discursive et font avancer la discussion. Il s'agira donc de présenter la mise en œuvre de l'approche didactique retenue, dont le but a été d'adapter au contexte scolaire les schémas interprétatifs initialement élaborés pour étudier la communication semi-formelle et endolingue dans les forums publics.

La recherche-action a été réalisée lors d'un cours de spécialisation proposé aux étudiants de Master FLE d'une université polonaise. Le sujet du cours portait sur l'analyse des interactions en ligne. Le corpus recueilli contient trois types de données : des extraits des forums de discussion publics et ouverts sélectionnés surtout par l'enseignant, les analyses des billets réalisées par les étudiants et les billets de forum qu'ils ont rédigés eux-mêmes. Dans le présent travail, nous nous référerons aux forums publics suivants:

- les forums proposés dans les sites médiatiques (notamment www.franceinfo.fr et www. madmoizelle.com) pour accompagner les articles relatifs à l'actualité politique et sociale, 
- l'espace «commentaires » accompagnant un blogue consacré aux questions environnementales (www.sortedevosconapts.blogspot.com).

Nous tenons à préciser que les billets rédigés par les étudiants n'ont pas été publiés : il s'agissait d'une activité d'entraînement, permettant d'analyser une «situation potentielle » de discussion dans le forum et dont le but était d'initier les étudiants à explorer et à intégrer les catégories d'observation de nature discursive, ainsi que de considérer leur utilité pour ce qui est de l'introduction à ce genre discursif. La démarche retenue suit celle de de Pietro, Schneuwly (2003) qui, pour préparer leurs élèves à de nouvelles pratiques discursives (genres argumentatifs tels l'exposé), ont travaillé à la fois sur les extraits de discours produits en dehors du contexte scolaire et sur les productions de leurs apprenants.

\section{3. ÉTAT DES RECHERCHES SUR LA DIMENSION FORMATRICE DES INTERACTIONS DANS LES FORUMS DE DISCUSSION PUBLICS ET OUVERTS}

Exploiter les forums publics dans le contexte d'enseignement/apprentissage d'une langue étrangère reste peu fréquent, ceci malgré le fait que leur potentiel pour ce qui est d'une pratique communicative authentique et variée est souvent souligné dans les recommandations adressées aux enseignants.

Parmi les recherches qui apportent des arguments en faveur des tâches misant sur les échanges avec les locuteurs natifs, il faut surtout mentionner les travaux de Hanna et de Nooy $(2003,2009)$ qui montrent comment et dans quelles conditions l'intégration des forums publics peut contribuer au développement de l'expression personnelle et argumentative. Dans le contexte polonais, il convient de citer le travail de Pędzisz (2009) : cette recherche décrit un scénario didactique visant le développement de la compétence interculturelle chez les étudiants de langue à partir d'une tâche les invitant à réagir à un billet de blogue, portant sur l'actualité socio-politique. L'objectif du projet était de mettre les apprenants en relation avec les contenus médiatiques et de les inviter à entrer en interaction avec les locuteurs natifs qui ont participé à cet échange.

Nous partageons la même perspective et nous reprenons, dans notre recherche, les éléments développés dans les travaux cités, notamment l'argumentaire présenté pour situer la dimension formatrice de toute interaction écrite et asynchrone engageant des locuteurs natifs. En effet, nous considérons que la valeur sous-jacente à la participation aux discussions en ligne se retrouve dans l'effort fourni par les locuteursapprenants pour maintenir le dialogue. Le succès pour les apprenants dépend alors de leur capacité à s'adapter aux normes socio-culturelles de mise dans la communauté donnée, mais également à construire une identité discursive qui leur permettra 
de proposer des billets qui trouveront une place dans le développement de la réflexion collective.

En adhérant à la définition du forum vu comme une discussion asynchrone et écrite réalisée dans un groupe restreint (Colin, Mourlhon-Dallies, 2004 ; MourlhonDallies, 2007), nous souhaitons privilégier, dans la manière de présenter les enjeux et la spécificité de la discussion aux étudiants, une perspective discursive. Ceci nous invite à mettre en relief les notions suivantes que nous considérons comme centrales pour notre travail :

\section{a. Le genre et le contrat de communication}

En définissant la discussion en ligne, nous insistons surtout sur le potentiel lié à la nature écrite et asynchrone des échanges pour ce qui est de la forme et de la qualité de la réflexion. Nous soulignons la dimension rationnelle de l'interaction, facilitée en plus par un accès aisé aux ressources supplémentaires disponibles sur Internet et nous insistons en même temps sur le nombre restreint de participants engagés dans la publication. En effet, le contrat de communication implique une modération des billets et offre le droit à la parole surtout à ceux qui acceptent une mise en scène qui favoriserait une expression rationnelle (Matuszak, 2007 ; Silva, 2015). Nous pouvons observer que les discussions les plus fécondes et longues sont celles où les locuteurs font un effort pour s'adapter aux mécanismes de la communication en ligne : ils pardonnent les maladresses possibles dans l'expression, évitent des attaques personnelles, restent focalisés sur le problème discuté et acceptent de soumettre leur interprétation à l'évaluation des autres. Le contrat de communication sous-jacent à la discussion en ligne implique donc, pour les locuteurs, le besoin de respecter les codes de la nétiquette, mais également le besoin de veiller au maintien de l'orientation argumentative dans leurs interventions.

\section{b. L'identité discursive et l'identité de penseur critique}

Ces deux notions sont essentielles dans le contexte de notre recherche puisque nous nous intéressons aux particularités de la discussion en ligne qui permettent de la considérer comme une situation d'apprentissage où les locuteurs présentent et explorent les arguments formulés dans l'espace public. Notre objectif est de mettre en relief l'identité de penseur critique : il s'agira, pour les apprenants, de prendre une posture réflexive qui leur permettra de se positionner par rapport aux arguments exprimés par les intervenants successifs et de considérer la validité et la pertinence des problèmes discutés, en tenant compte à la fois de l'intention des locuteurs et des standards de la pensée critique. Nous suivons ici le raisonnement de Doury et Maroccia (2007) et nous considérons que l'éthos de penseur critique devient donc une « ressource discursive » pour mettre en jeu la légitimité du point de vue avancé. 
c. L'orientation argumentative et les contributions des locuteurs au travail collégial de réflexion

Comme le remarque Amossy, «le discours cherche toujours à avoir un impact sur son public. Il s'efforce souvent de le faire adhérer à une thèse : il a alors une visée argumentative. Mais il peut aussi, plus modestement, chercher à influencer des façons de voir et de sentir : il possède dans ce cas une dimension argumentative » (2012, p. 3). Nous retenons cette distinction dans notre travail afin d'insister sur les relations entre les intentions des locuteurs et le discours produit puisqu'elles peuvent être exploitées dans la réflexion sur la place que chaque commentaire est censé jouer dans le déroulement de l'interaction. Pour ce qui est des analyses de la discussion en ligne, la mise en relief de la nature argumentative de l'interaction permet également d'insister sur le caractère négocié des processus de compréhension et de coconstruction du sens et de montrer que c'est la lecture du fil de discussion par les participants successifs qui décide de la qualité de la réflexion qui s'y forme.

La mise en relief de l'approche discursive dans la manière d'appréhender les interactions dans les forums publics et ouverts invite ainsi à considérer aussi bien la lecture des billets que leur rédaction comme une activité ayant un grand potentiel pour chaque apprenant, ceci aussi bien pour le développement de sa réflexion citoyenne que de sa compétence discursive.

\section{COMPTE RENDU DE RECHERCHE : ÉLABORER UN OUTIL DIDACTIQUE}

Les résultats obtenus dans la recherche seront présentés dans deux sections successives qui correspondent à nos deux questions de recherche spécifiques, telles qu'elles ont été formulées dans notre projet.

\subsection{PRÉCISER LES OBJETS D'OBSERVATION POUR AIDER LES LOCUTEURS À INTÉGRER LA DISCUSSION DANS LES FORUMS}

Nous avançons dans notre travail que les catégories d'observation issues de l'analyse du discours offrent aux locuteurs s'initiant à la discussion dans le forum une perspective d'analyse qui facilite la lecture de billets et la prise de parole dans l'interaction. Le forum n'est plus considéré comme un espace désordonné ou chaotique et la lecture des billets devient - aux dires des apprenants eux-mêmes - une expérience souvent enrichissante, qui offre une inspiration et qui nourrit leur réflexion. La valeur de cette expérience est particulièrement prégnante pour la lecture des forums en langue étrangère et les apprenants soulignent que la lecture des billets 
qui prend en compte les enjeux discursifs permet de mieux comprendre comment le problème est construit et négocié, ainsi que de se positionner plus efficacement face aux participants et à leurs commentaires.

Nous présenterons ci-dessous une grille élaborée dans le cadre de notre recherche-action. Elle insiste sur cinq catégories d'observation : forum en tant que dispositif ; normes génériques sous-jacentes à la rédaction; identité discursive des locuteurs ; identité de penseur critique ; orientation argumentative du billet. Les visées d'observation formulées dans le cadre de notre recherche mettent ainsi en relief les particularités du genre, de même que les éléments constitutifs du contrat de communication permettant aux locuteurs de partager les finalités et d'accorder une même valeur à cette expérience de réflexion citoyenne. Détaillées à travers une série de questions, elles nous ont servi à expliciter nos objectifs d'enseignement/ apprentissage.

Tableau 1. Les catégories d'observation élaborées dans le cadre de la recherche-action présentée

Observer l'interaction dans les forums ouverts et publics pour intégrer la communauté en ligne

1. Observer le forum comme un dispositif de communication électronique

Quelle relation les participants entretiennent-ils avec l'auteur de l'article commenté ? L'auteur intervient-il dans le forum ?

Le forum repose-t-il sur une modération? Par qui est-elle réalisée ? Comment est-elle vécue par les participants?

De quel type de forum s'agit-il (accessible au grand public ou à une communauté restreinte ; la publication y est rapide ou bien offerte seulement aux enregistrés, etc.) ?

Le forum a-t-il une orientation plutôt communautaire (p. ex. les commentaires dans un blogue) ou argumentative (p. ex. les forums des sites médiatiques) ?

2. Observer la conformité du billet aux normes génériques (celles de la discussion asynchrone et écrite, réalisée dans un groupe restreint)

À qui s'adresse le billet? L'auteur vise-t-il à préciser le destinataire ?

Vers quelle finalité (évaluer, prouver, nier, capter, etc.) le billet est-il orienté ? Cette finalité est-elle facile à saisir?

Dans quelle mesure le billet respecte-t-il les conventions génériques (notamment la dimension rationnelle du raisonnement et le respect des standards de la pensée critique) ?

Comment les locuteurs gèrent-ils les conflits interpersonnels ?

3. Observer l'identité des locuteurs engagés dans la communication en ligne

Qui sont les intervenants ? Comment expliquent-ils leur décision de prendre la parole ? (p. ex. un citoyen, un lecteur qui s'intéresse à l'actualité, etc.)

À qui s'adressent-ils ? Comment construisent-ils l'identité de leur(s) interlocuteur(s) ?

Qui, selon les intervenants, constitue le public de ces sites ? Qui peut être intéressé par ces billets ? Quelle est la réaction attendue de la part des lecteurs-locuteurs?

4. Endosser l'identité discursive de penseur critique pour s'exprimer et pour intégrer la communauté discursive

Le locuteur se réfère-t-il aux sources d'informations extérieures (presse, sites Internet) pour appuyer son argument? 
Observer l'interaction dans les forums ouverts et publics pour intégrer la communauté en ligne

Le locuteur respecte-t-il les standards de la pensée critique dans son raisonnement ? Veille-t-il à la cohérence, à la précision, à la clarté de son raisonnement ?

Quand et sous quelles conditions est-il prêt à modifier son raisonnement face aux réactions des autres ?

Le locuteur accepte-t-il l'effort d'évaluer le raisonnement de l'autre?

Le locuteur accepte-t-il l'effort d'identifier et d'expliquer les incohérences, les points de désaccord éventuels dans les interventions précédentes?

5. Saisir l'orientation argumentative du billet en tant que facteur influant sur le déroulement de l'interaction

En quoi se ressemblent et en quoi varient les positions face au sujet?

Quel est l'impact de chaque billet pour le déroulement de l'interaction?

La chronologie de la publication a-t-elle un impact sur les objectifs et la forme des billets successifs?

Quels sont les billets qui provoquent la réaction des autres ? De quoi dépend cette réaction?

Les recherches sur l'interaction dans les forums, telles qu'elles sont réalisées dans le champ de l'analyse du discours, offrent de nombreuses pistes pour saisir la logique et les enjeux des discussions dans les espaces ouverts et publics. Nous situons la valeur de l'observation outillée que nous proposons dans la mise en relief des visées suivantes :

- observer comment les locuteurs interprètent leurs billets successifs, donc lier l'efficacité et la qualité de l'argumentation aux conditions situationnelles;

- observer les mécanismes argumentatifs pour identifier les conduites de la pensée critique qui sous-tendent l'argumentation rationnelle;

- observer les éléments constitutifs de la compétence de communication attendue chez un locuteur compétent, afin de proposer un profil de compétence qui insistera sur l'identité discursive de penseur critique ;

- développer les stratégies de lecture critique et mettre en valeur la lecture comme une situation d'apprentissage permettant au lecteur de mieux comprendre comment les arguments sont construits dans l'espace public et repris ensuite dans les raisonnements individuels.

Notre démarche offre donc une approche discursive censée contribuer à l'explicitation des particularités du genre et à l'opérationnalisation des conduites rédactionnelles pouvant faciliter un engagement efficace aux locuteurs dans une réflexion collégiale en ligne. Nous illustrerons, dans la section suivante, comment les catégories d'observation proposées ont été utilisées dans le cadre de notre recherche-action portant sur l'initiation des locuteurs au nouveau genre discursif. Nous tenons à rappeler que la démarche entreprise appartient à l'étape exploratoire : il s'agira d'illustrer comment l'enseignant peut puiser dans le répertoire de questions élaborées pour - d'un côté - préciser la description en la rapportant aux exemples concrets et - de l'autre - tester la lisibilité des catégories auprès de ses étudiants. 


\subsection{EXPLOITER LES NOTIONS ÉLABORÉES AU SEIN DE L'ANALYSE DU DISCOURS DANS L'OBSERVATION DE L'ACTIVITÉ INTERPRÉTATIVE ET RÉDACTIONNELLE DES LOCUTEURS}

Dans le scénario proposé, nous avons demandé aux étudiants de rédiger des billets qui pourraient être publiés dans les forums de discussions publics français. En tant qu'enseignante, nous avons fourni une liste de forums pour orienter la recherche des étudiants, tout en les encourageant à explorer individuellement d'autres espaces publics de discussion du même type : les apprenants ont toutefois préféré choisir les sites dans la liste proposée. Étant donné qu'il s'agissait d'une activité d'exploration, les billets rédigés par les étudiants n'ont pas été publiés dans les forums retenus. Les locuteurs ont néanmoins précisé la place dans le fil de discussion qui leur était destinée : selon les indications fournies, tous les billets présentés étaient censés paraître en tant que le dernier message dans la suite de billets déjà publiés sur le site au moment de la réalisation de la tâche.

Nous avons choisi quatre billets pour illustrer en quoi la prise en compte des phénomènes discursifs au travers des catégories d'observation proposées contribue à initier les apprenants à un nouveau genre discursif - celui des discussions dans les forums publics et ouverts. Les exemples choisis montreront comment le feedback apporté par l'enseignante permet de mettre en relief et d'expliciter les objectifs d'une évaluation portant sur l'authenticité de chaque intervention, tout en tenant compte du contexte de la séquence dans laquelle le billet s'inscrit.

\subsubsection{L'EXEMPLE 1}

Le premier billet que nous soumettons à notre analyse a été proposé pour commenter un article de blogue, dont la thématique est consacrée aux questions du développement équitable. Son auteure se présente comme suit : «Je suis une maman, un être humain qui milite pour démontrer que consommer raisonnablement, vivre simplement, et avec respect, tout en restant intégrée dans la société, est possible ». L'article du blogue commenté par l'étudiante s'intitule «Vis ma vie de blogueuse en vacances : la mer ${ }^{1} \gg$. L'étudiante intervient en tant que septième commentatrice, les billets précédents ont été publiés en l'espace de cinq jours en juin 2016.

Les touristes sont en grande partie responsable des déchets que l'on trouve au bord de la mer. Chaque fois que je me déplace je ramasse systématiquement mes déchets, si tout le monde faisait pareil il n'y rien à ramasser. La conscience écologique est importante pour nous tous et nous nous engageons à contribuer au développement durable. Plus d'inquiétude pour l'espace commun !?

\footnotetext{
${ }^{1}$ http://www.sortezdevosconapts.com/2016/08/vis-ma-vie-de-blogueuse-en-vacances-la-mer.html

${ }^{2}$ Dans les exemples fournis, nous rapportons fidèlement les billets de nos étudiantes.
} 
L'activité de la locutrice respecte le principe d'interaction dont parle Charaudeau (1993), puisqu'elle adopte efficacement une posture lui permettant d'intégrer le groupe : comme les locuteurs précédents, cette étudiante intervient pour donner son avis, mais elle ne met pas en doute ni ne critique le raisonnement de la blogueuse. Elle expose son propre raisonnement et le soumet à l'évaluation du groupe, en calquant en ceci son comportement sur celui des intervenants précédants. En endossant l'identité d'une touriste - vacancière soucieuse de l'environnement et veillant à ne pas le dégrader, la locutrice se réfère explicitement aux normes et aux valeurs qu'elle identifie comme partagées par le groupe. En ce qui concerne la place que le billet occupera dans le déroulement de l'interaction (principes d'influence et de régulation, Charaudeau 1993), il remplit les mêmes objectifs que les billets précédents : le fait de prouver son attitude par des gestes concrets qui y sont décrits (le billet contient un exemple personnel) légitime l'auteure dans son appartenance à la communauté. En effet, l'étudiante souligne avoir les mêmes préoccupations et le jugement implicite qu'elle fournit est censé valider les propos de la bloggeuse. Le fait de donner ses motifs est une stratégie pour intégrer une communauté discursive de lecteurs représentant une attitude écologique similaire.

Lors d'un travail pédagogique, l'analyse de ce billet a relevé plusieurs points permettant de mieux cerner la nature de l'argumentation en ligne. Tout d'abord, il nous a été possible de rappeler que l'intégration d'un espace de discussion ouvert repose sur différentes stratégies argumentatives, les trois conduites essentielles étant la justification, l'explication ou la démonstration (Forget, 2015). Dans l'espace de discussion observé, la plupart des billets prennent une orientation plutôt démonstrative (une solution est possible, et donc décrite dans le billet) que justificative, qui pourrait, par exemple, viser à légitimer la prise de position adoptée face au problème de la propreté des espaces communs. Cette attitude permet aux membres de la communauté de consolider le groupe, mais, du point de vue discursif, elle peut avoir des conséquences sur la forme de l'argumentation, notamment sur sa rigueur. Comme cet exemple le montre, l'orientation démonstrative dans le raisonnement peut amener les locuteurs à proposer un raisonnement qui repose sur un apparent «sens commun », où il est difficile de voir des choses différemment. Aux yeux de l'étudiante, la solution au problème observé semble s'imposer : sa finalité est donc de confirmer la faisabilité du choix de ceux qui ne laissent pas leurs déchets dans la nature et de conforter la blogueuse et les commentateurs dans leurs convictions.

L'exemple choisi permet de rappeler aux étudiants que l'interaction prend une orientation critique et rationnelle seulement lorsque les locuteurs se positionnent par rapport aux idées véhiculées dans l'interaction. La prise de position explicite est une stratégie qui mobilise les locuteurs à réfléchir à leurs ressources, donc à adopter une attitude critique face à leur raisonnement et au sujet discuté. Dans le billet analysé, la locutrice fait savoir qu'elle partage la perspective de la blogueuse : elle ne tend pas à préciser davantage le problème, à s'assurer de la cohérence ou bien de la complétude du raisonnement développé à travers les interventions successives 
puisqu'elle décide de ne pas se positionner face aux billets publiés. Le billet n'ouvre donc pas de nouvelles pistes dans le développement de la réflexion collective. Le travail didactique offre une occasion pour réfléchir aux stratégies discursives qui auraient pu renforcer la dimension plus argumentative de l'interaction.

\subsubsection{L'EXEMPLE 2}

Les deux billets suivants (exemples 2 et 3 ) se réfèrent au même article publié sur le site www.madmoizelle.com. L'article «Les voitures de métro réservées aux femmes, une idée anti-harcèlement pas si nouvelle » a été rédigé par Mimy, une journaliste du magazine, le 3 avril 2017. Vingt-sept commentaires ont été publiés en réaction à ce texte.

Merci bien pour un article super intéressant. Le harcèlement est une vraie menace où que ce soit. Suis d'accord avec l'auteur - voiture de métro réservée aux femmes me semble pas un moyen de prévention trop efficace. Il vaut mieux d'investir cet argent aux campagnes de sensibilisation. D'ailleurs bravo Cam Clash pour montrer dans un de ses vidéos avec caméra cachée harcèlement sexuel dans les transports en commun (vous en avez déjà parlé il y a un mois). Faut que les gens prennent la conscience et qu'ils aient pas peur de réagir quand ils voient des abus dans la rue!

Le billet cité offre plusieurs pistes pour une analyse didactique, permettant de mettre en relief deux notions discursives : celles de communauté et d'identité discursive.

On y retrouve de nombreux indices permettant de voir l'effort que fait la locutrice pour marquer son appartenance à la communauté de pratique, dont les membres s'inspirent réciproquement. C'est par exemple la référence aux articles publiés antérieurement sur le site qui permet à l'étudiante de se présenter comme une lectrice régulière. Elle insiste ainsi sur les centres d'intérêts partagés par les lectrices et les journalistes et apprécie le rôle que le site joue pour le dialogue social : les rédactrices informent sur des événements et des initiatives importants, se révoltent contre certains comportements, signalent des solutions. De plus, la lecture du site est ici présentée à la fois comme un moyen d'apprendre de nouvelles choses et comme une expérience permettant à la lectrice de consolider ses convictions personnelles et d'y puiser des arguments pour évaluer les événements et les situations qu'elle discute au quotidien.

L'identité que la locutrice endosse est donc celle d'une citoyenne engagée dans plusieurs débats sociaux, adaptant une attitude de recherche de solutions face aux problèmes présentés dans les articles. Elle se présente aussi comme une lectrice qui apprécie le site comme source d'inspiration, permettant de s'informer et de réfléchir davantage. Le forum ouvert devient ainsi un espace d'intégration sociale offrant - grâce à la forme collégiale de la réflexion - des moyens pour former une attitude critique. 


\subsubsection{L'EXEMPLE 3}

Comme dans l'exemple précédent, l'auteur du billet proposé ci-dessous se positionne par rapport au problème présenté dans l'article «Les voitures de métro réservées aux femmes, une idée anti-harcèlement pas si nouvelle» publié sur le site www.madmoizelle.com

L'idée de protéger les femmes de l'agression masculine est bien juste, je ne pense pourtant pas que cette manière de la réalité est parfaite. Les wagons destinés aux femmes me font penser à la discrimination plutôt qu'à la protection - comme si c'étaient les femmes qui provoquaient à un acte d'agression, il faudrait donc les séparer de la foule. Au lieu de créer de tels espaces, je souhaiterais plutôt que les femmes n'aient pas peur de parler de ce qui se passe chez elles et qu'elles ne soient pas considérées comme coupables alors qu'elles sont en réalité victimes - les agresseurs ne se sentiraient plus alors si innocents.

L'étudiante semble répondre à la question que la rédactrice formule dans le dernier paragraphe de son texte : «Je préfère personnellement voir le gouvernement allier des fonds, du personnel et des campagnes de sensibilisation à ce sujet que de me parquer dans une voiture réservée aux femmes... mais c'est mon avis. Quel est le vôtre ? $^{3}{ }$. Cependant, une lecture attentive de l'article et du billet permet de constater que le billet ne s'inscrit pas à vrai dire dans la problématique soulevée par la journaliste. On peut en effet relever un changement de la perspective dans laquelle l'idée est discutée: la journaliste s'inspire de l'idée des wagons réservés aux femmes pour reconsidérer cette solution par rapport au problème défini dans l'article comme un harcèlement sexiste, tandis que l'étudiante, dans son billet, revendique elle aussi la nécessité d'insister sur la responsabilité des hommes mais, en même temps, elle déplace le contexte pour parler d'une situation des violences domestiques.

L'exemple rapporté permet d'illustrer un mécanisme fréquent dans les discussions informelles où les locuteurs restent moins attentifs à leurs billets respectifs et où leur intervention peut être motivée surtout par une décision spontanée de «s'exprimer», de «réagir». La progression se fait alors par une accumulation de billets offrant des prises de positions personnelles. Compte tenu des objectifs de notre projet didactique, l'analyse de ce billet invite à introduire les notions de dialogue réflexif et d'argumentation rationnelle, telles qu'elles sont décrites dans les travaux portant sur les interactions collaboratives visant à résoudre un problème (Garrison, 1991 ; Newman et al. 1995). Ces auteurs considèrent que ce sont les conduites d'évaluation qui permettent aux locuteurs de renforcer l'orientation argumentative de l'interaction et ils décrivent les conduites de la pensée critique censées

\footnotetext{
${ }^{3}$ L'extrait vient du dernier paragraphe de l'article «Les voitures de métro réservées aux femmes, une idée anti-harcèlement pas si nouvelle » publié par Mimy le 3 avril 2017 sur le site http://madmoi zelle.com URL : http://www.madmoizelle.com/metro-reserve-femmes-manon-bril-752221
} 
assurer la concentration sur le sujet et sur la pertinence des interventions par rapport au problème défini. Étant donné que la journaliste et l'étudiante mettent chacune un autre problème social au centre de leur réflexion, une analyse de ce billet qui tiendra compte de l'article auquel le billet se réfère, offre une occasion de réfléchir aux contenus d'une intervention qui serait explicitement orientée vers l'exploration critique des thèses proposées par la journaliste.

\subsubsection{EXEMPLE 4}

Il s'agit ici d'une intervention proposée dans l'espace de commentaires sur le site d'information www.franceinfo.fr qui accompagne l'article de la rédaction publié le 30 mars 2017 et intitulé «États-Unis : Ivanka Trump, conseillère de son père, devient employée fédérale ». Le billet propose une réaction dans un fil de discussion comptant douze commentaires. L'article en question portait sur un sujet qui n'était plus d'actualité au moment de la tâche (fin avril 2017), le fil de discussion étant déjà éteint.

Laissez la tranquille, cette femme. C'est vrai que si on possède bcp d'argent on peut même travailler gratuitement mais comme un proverbe connu le dit «Chacun son métier les vaches seront bien gardées ».

Le billet suivant nous a permis de mettre en avant, à part la notion d'identité discursive, aussi celle de politesse dans les échanges électroniques. L'interaction en ligne est une situation de communication impliquant des relations symétriques, régulées - au niveau social - par une nétiquette et soumises en plus à une modération plus ou moins rigoureuse (Silva, 2015). Le billet proposé a l'avantage d'exprimer un point de vue personnel, mais sa structure rédactionnelle rend l'interprétation de l'argument ambiguë : il est en effet difficile de préciser de façon univoque la position de l'étudiante face au problème discuté. La première phrase, de même que le proverbe cité, laissent supposer une attitude plutôt positive par rapport à la décision de Ivanka Trump de ne pas être rémunérée pour son travail. En même temps, cependant, l'organisation des idées à l'intérieur du billet (« c'est vrai que (...) mais comme $(\ldots) \gg)$ brouille l'interprétation et l'étudiante elle-même a confirmé notre difficulté de lecture. Lors de l'analyse de ce billet, notre attention a donc été surtout orientée vers la notion de stratégies conversationnelles directes et vers l'impact que l'expression de son jugement peut avoir sur la manière dont les locuteurs construisent leurs relations. Nous voulions montrer que la stratégie choisie par l'étudiante risque de provoquer un conflit avec les intervenants précédents qui peuvent se sentir offensés par ce rejet ouvert de leur questionnement et donc - de manière plus générale - par le refus de légitimer le bien-fondé de la controverse éthique dont ils discutent. 
Notre intention dans la présente section a été d'illustrer la démarche d'application pour la grille d'observation élaborée dans le cadre de la recherche-action et de mettre en relief les enjeux et les avantages d'une analyse orientée vers les aspects discursifs. Le fait de demander aux étudiantes de rédiger un billet qu'elles pourraient publier dans le fil de discussion de leur choix nous a permis - à travers les catégories d'observation retenues - de présenter certains particularités génériques de la discussion en ligne et de mieux cerner les conditions de la participation réussie. A cette étape exploratoire, notre pratique enseignante confirme qu'il est possible d'opérationnaliser certaines notions faisant partie de l'appareil de l'analyse du discours dans un travail didactique : le travail d'observation et d'analyse proposé offre aux locuteurs-apprenants des outils qui permettent de mieux saisir et d'expliquer le rapport entre le billet proposé et les choix communicatifs, relationnels et cognitifs qui le sous-tendent.

\section{CONCLUSIONS À L'ISSUE DE NOTRE RECHERCHE-ACTION}

Le travail réalisé confirme nos hypothèses initiales quant au potentiel explicatif des catégories d'observation ancrées dans la perspective discursive et appliquées pour décrire et analyser les interactions en ligne. Tout d'abord, le recours à l'appareil notionnel de l'analyse du discours permet de mettre en relief les particularités formelles relatives aux discussions dans les forums publics et ouverts et de situer ce genre dans un continuum des discours médiatiques et argumentatifs. Ensuite, le scénario didactique proposé fait découvrir aux apprenants le potentiel didactique de ces espaces de communication puisque les questions contenues dans la grille élaborée mettent en relief l'intention et la valeur donnée à cette expérience par les locuteurs engagés dans l'échange et contribuent à expliciter des règles qui gèrent les pratiques discursives des communautés virtuelles. Ce regard est souvent nouveau pour les apprenants, habitués à privilégier dans leur évaluation la correction linguistique et les règles de politesse, au détriment des enjeux sociaux et institutionnels.

Les résultats obtenus invitent à donner une suite à la démarche proposée : pour les étudiantes engagées dans notre projet, il serait sans doute formateur d'observer comment les billets qu'elles rédigent peuvent être interprétés par les membres de la communauté discursive auxquels elles s'adressent. Cette étape contribuerait à une meilleure validation de la pertinence du modèle générique que nous avons construit dans notre recherche, notamment pour ce qui est de la manière dont nous définissons le contrat de communication censé assurer un déroulement fluide et cohérent de l'interaction et nous schématisons les relations entre les identités discursives des locuteurs et les la manière dont nous saissons finalités de l'échange. 


\section{BIBLIOGRAPHIE}

Amossy, R. (2012). L'argumentation dans le discours. Paris : Armand Colin.

Charaudeau, P. (1993). Le contrat de communication dans la situation classe. In J. F. Halté (réd.), Inter-Actions (pp. 121-135). Metz: Presses Universitaires de Metz. URL: http://www.patrickcharaudeau.com/Le-contrat-de-communication-dans.html

Charaudeau, P., Mangeneau, D. (réd.) (2002). Dictionnaire d'analyse du discours. Paris : Seuil.

Colin, J.-Y., Mourlhon-Dallies, F. (2004). Du courrier des lecteurs aux forums de discussion sur l'internet : retour sur la notion de genre. Les Carnets $d u$ Cediscor, 8. URL : https://cediscor.revues. org/707

De Pietro, J.-F., Schneuwly, B. (2003). Le modèle didactique du genre : un concept de l'ingénierie didactique. Les cahiers THÉODILE, 3, 27-52.

Doury, M., Lefébure, P. (2006). 'Intérêt Général', 'Intérêts Particuliers'. La Construction de l'Ethos dans un Débat public. Questions de communication, 9. URL : https://questionsdecommunication. revues.org/7922

Doury, M., Maroccia, M. (2007). Forum Internet et courrier des lecteurs : l'expression publique des opinions. Hermès, 47 (1), 41-50. URL : http://www.cairn.info/revue-hermes-la-revue-2007-1-page41.html

Forget, M.-H. (2015). Les conduites de justification : un savoir à transposer des usages. Bellaterra Journal of Teaching \& Learning Language \& Littérature, 8 (1), 46-59.

Garrison, D. R. (1991). Critical thinking and adult education: a conceptual model for developing critical thinking in adult learners. International Journal of Lifelong Education, 10 (4), 287-303.

Hanna, B. E., de Nooy, J. (2003). A funny thing happened on the way to the forum: electronic discussion and foreign language learning. Language Learning \& Technology, 7 (1), 71-85. URL : http://llt. msu.edu/vol7num1/hanna

Hanna, B. E., de Nooy, J. (2009). Learning Language and Culture Via Public Internet Discussion Forums. New York : Palgrave Macmillian.

Matuszak, C. (2007). L'environnement discursif des forums politiques : le cas des forum d'organisation politiques marginales. Études de communication, 30. URL : https://edc.revues.org/510.

Mourlhon-Dallies, F. (2007). Communication électronique et genres du discours. Glottopol. Revue de sociolinguistique en ligne, 10, 11-23. URL : http://glottopol.univ-rouen.fr/telecharger/numero_10/ gpl10_01mourlhon.pdf

Newman, D. R., Webb, B., Cochrane, C. (1995). A content analysis to measure critical thinking in faceto-face and computer supported group learning. Interpersonal Computing and Technology, 3(2), 56-77.

Pędzisz, J. (2009). Blog jako narzędzie wspierające rozwój kompetencji dyskursywnej. Neofilolog, 33, $101-112$.

Silva, M. T. (2015). What do users have to say about online news comments ? Readers' accounts and expectations of public debate and online moderation: a case study. Participations. Journal of Audience \& Reception Studies, 12 (2), 32-44. URL : http://www.participations.org

Thiault, F. (2009). Des méthodes combinées pour une analyse communicationnelle d'une liste de discussion professionnelle. Études de communication, 33. URL : http://edc.revues.org/1096 